DOI: 10.1002/aenm.((please add manuscript number))

Submitted to

ENERGY

Article type: Full Paper

\title{
A Systematic Approach to the Design Optimization of Light Absorbing Indenofluorene Polymers for Organic Photovoltaics
}

James Kirkpatrick, * Christian B. Nielsen, * Weimin Zhang, Hugo Bronstein, R. Shahid Ashraf, Martin Heeney, and Iain McCulloch

Dr. J. Kirkpatrick

Mathematical Institute, University of Oxford, 24-29 St. Giles, Oxford OX1 3LB (UK)

E-mail: kirkpatrick@maths.ox.ac.uk

Dr. C. B. Nielsen, Dr. W. Zhang, Dr. H. Bronstein, Dr. R. S. Ashraf, Dr. M. Heeney, Prof. Iain McCulloch

Department of Chemistry and Centre for Plastic Electronics, Imperial College London, London SW7 2AZ (UK)

E-mail: c.nielsen@imperial.ac.uk

Keywords: conjugated polymers; DFT calculations; molecular simulations; organic photovoltaics; solar cells

The synthesis and optimization of new photovoltaic donor polymers is a time consuming processes. Computer based molecular simulation can narrow the scope of materials choice to the most promising ones, by identifying materials with desirable energy levels and absorption energies. In this paper, we present such a retrospective analysis to a series of fused aromatic push-pull copolymers. We demonstrate that molecular calculations do indeed provide good estimates of the absorption energies measured by UV-Vis spectroscopy and of the ionization potentials measured by photoelectron spectroscopy in air. Comparing measured photovoltaic performance of the polymer series to the trend in efficiencies predicted by computation confirms the validity of this approach. 


\section{Introduction}

Submitted to MATERIALS

Solar energy has the potential to meet most of the world's demand for electricity, and research into solar cells has consequently received much attention over the last decade. Organic photovoltaic (OPV) materials, either in the form of small molecules, oligomers or polymers, hold great potential for solar cell applications due to their ease of processing, their high optical absorptivity and the ease with which electronic properties can be tailored through molecular design. ${ }^{[1-5]}$

Three distinct steps are necessary to generate energy from solar radiation: i) photons must be absorbed, ii) the resulting excited states must be converted into free charges, and iii) these free charges must be collected. The efficiency and energy losses involved in each of these steps will determine the overall efficiency of a solar cell. For example, a material which has too large a band gap will have poor absorption at the long wavelength region of the solar spectrum, resulting in low charge generation which manifests as a low short circuit current $\left(\mathrm{J}_{\mathrm{sc}}\right)$ in a photovoltaic device. In an organic solar cell, excitons are separated by the difference in electron affinity between electron donors and acceptors. This difference must be large enough to ensure the quenching of the excitons. There is a limit to how big this difference can be as the open circuit voltage $\left(\mathrm{V}_{\mathrm{oc}}\right)$ of the device depends on the difference between the highest occupied molecular orbital (HOMO) of the donor and the lowest unoccupied molecular orbital (LUMO) of the acceptor. Increasing the difference in electron affinities of electron donor and acceptor, whilst keeping the band gap of the donor the same (i.e. increasing the electron affinity of the acceptor), will therefore reduce the maximum voltage available from the device. It should be noted that energy level alignment is not in itself sufficient to ensure the generation of free charges: exciton diffusion lengths are short and therefore it is important that the blend morphology of the donor and acceptor in a bulk heterojunction $(\mathrm{BHJ})$ device is such as to ensure that a large fraction of the generated excitons are quenched at the interface. It should also be noted that excitations can be quenched not 
Submitted to MATERIALS

only by producing free charges but also by producing triplets and charge transfer states. The final step, charge transport, is crucial to high efficiencies: if transport is a dynamic bottleneck the overall efficiency will decrease ${ }^{[6]}$ if the path to the electrodes is tortuous it is fair to assume that recombination will play a bigger role.

It is clear from these simple considerations that predicting the performance of a new photovoltaic donor material is a complicated challenge, in particular because the morphology of a binary blend with the acceptor material is unpredictable, and very sensitive to processing conditions. Even defining the "ideal" morphology is a balance of compromises: the pristine phases should be 1) not too coarsely separated otherwise exciton quenching would be inefficient but also 2) not too finely intermixed or recombination would become dominant. In addition to the process of phase separation, the subsequent morphology of each phase is also very important for achieving high mobilities, as illustrated by studies on the regioregularity of poly(3-hexylthiophene) (P3HT) and on the influence of annealing. ${ }^{[7-10]}$ Many examples exist in the literature which illustrate that modification of the heterojunction morphology has a large effect on the solar cell efficiency. This is most frequently displayed by varying the solubilising side-chains of the polymer backbone, which can affect the device performance to great extents. ${ }^{[11,12]}$ The molecular weight has likewise been shown to be of importance for the OPV properties. ${ }^{[13,14]}$ For a poly $\left(o\right.$-phenylene vinylene) derivative (MDMO-PPV),${ }^{[15]}$ the change of solvent from toluene to chlorobenzene afforded a large increase in efficiency, and in the case of a cyclopentadithiophene-benzothiadiazole copolymer, ${ }^{[16]}$ the use of an additive resulted in higher efficiencies. Similarly, the right combination of solvent and additive has been found to be of importance. ${ }^{[17]}$ It is clear from these literature examples that a successful OPV donor material might not achieve its full potential efficiency unless significant tuning of processing conditions is carried out to optimize the morphology. One of the advantages of organic materials is that they can be molecularly tailored to modify their properties. In light of the previous observations, this opportunity is also a potential draw-back as each new material 


\section{Submitted to MATERIALS}

may require unique device fabrication conditions, which are arrived at as a result of significant experimental trial and error, and hence progress can be slow.

Approximate guidelines and models of the maximum potential efficiency of a material are therefore important to select which materials should be selected for optimization. The screening of materials for device applications must both reduce false negatives (materials with good potential efficiencies should not be disregarded) and must be simpler to implement than the laborious steps of device optimization. Following from the work of Brabec and collaborators, we employ first a purely theoretical model and subsequently an empirical model, which takes into account the measured frontier energy levels, to gauge the maximum achievable efficiency in a photovoltaic device. ${ }^{[18]}$ In these models, the short circuit current is determined by the band gap of the polymer alone (either the calculated or the measured value) and the open circuit voltage by the difference between the donor HOMO and the acceptor LUMO; this model is described more fully in the methods section. The experimental band gaps and HOMO levels of the donors that we have designed and synthesized for this study can be easily measured using UV-vis spectroscopy and ultraviolet photoelectron spectroscopy in air (PESA). The potential for good charge transport in the blends is evaluated by measuring the field-effect transistor (FET) mobilities of the pristine donor materials. FET mobilities are perhaps a less appropriate indicator of charge transport under PV conditions than time of flight mobilities because they are in-plane measurements under high charge densities, however they are still a valid measure of a material's charge mobility. Additionally FET measurements fulfill the second requirement of a good screening method: they are easy to implement, unlike time of flight measurements which can require laborious sample preparation to ensure thick enough samples.

The additional advantage of focusing on band gap, HOMO level and mobility is that these properties can easily be related to the chemical structure of a polymer through both chemical intuition and quantum chemical calculation. The starting point for this investigation is the 
Submitted to MATERIALS

alternating poly(indenofluorene-co-triarylamine) (IF-TAA) depicted in Figure 1. Our previous studies have shown that IF-TAA is a good hole transport material due to the extended conjugation of the indenofluorene (IF) unit. ${ }^{[19]}$ Keeping this bridged molecular design motif for the main polymer backbone unit is expected to promote good mobilities with a range of co-repeat units. Our design goal is to reduce the band gaps of the second generation analogous polymers whilst ensuring high mobilities and without raising the HOMO levels to a point where the maximum potential efficiencies are reduced. Another consideration we have taken into account is the fact that the triarylamine (TAA) unit disrupts intermolecular $\pi$ stacking, which leads to amorphous polymers. The replacement of TAA with more coplanar units will help promote crystallinity and possibly also phase segregation, which can be advantageous for good performance in BHJ solar cells.

\section{Methods}

\subsection{Maximum efficiency model}

In order to approximate the maximum possible efficiency, three quantities must be estimated: the $\mathrm{J}_{\mathrm{sc}}$, the $\mathrm{V}_{\mathrm{oc}}$ and the fill factor. We have estimated the $\mathrm{V}_{\mathrm{oc}}$ as LUMO - HOMO -0.3 , following literature precedence. ${ }^{[18]}$ The short circuit current is obtained by assuming that all solar flux below the absorption edge is absorbed and converted to charge carriers by a quantum efficiency $\Phi$. In order to ensure that carriers are only generated if the LUMO of the donor $\left(\mathrm{LUMO}_{\mathrm{D}}\right)$ is greater than the LUMO of the acceptor $\left(\mathrm{LUMO}_{\mathrm{A}}\right)$, we model the quantum efficiency with the following equation:

$$
\begin{gathered}
\Phi=0.8 \exp \left(\frac{L U M O_{D}-L U M O_{A}}{k T}\right) \text {, if } L U M O_{A}<L U M O_{D} \\
\Phi=0 \text {, if } L U M O_{\mathrm{A}}>L U M O_{\mathrm{D}}
\end{gathered}
$$


As in the original work of Brabec et al., the fill factor is simply assumed to be $0.65{ }^{[18]}$ The theoretical frontier energy levels are calculated as outlined below, while the experimental value for the LUMO of the acceptor is assumed to be that of $\mathrm{PC}_{61} \mathrm{BM}\left([6,6]-\right.$ Phenyl- $\mathrm{C}_{61}{ }^{-}$ butyric acid methyl ester) at $-3.7 \mathrm{eV} .^{[20]}$ The experimental HOMO energy level of the donor material is measured by photoelectron spectroscopy in air (PESA) and the S1 energy corresponds to the onset of optical absorption in the solid state as measured by UV-vis.

\subsection{DFT calculations}

Calculations of the band gaps and HOMO levels of the various donors have been carried out using the hybrid density functional B3LYP and a double zeta split basis set with polarization functions. The exchange functional B3 being the hybrid method proposed by Becke ${ }^{[21]}$ that includes a mixture of Slater exchange, ${ }^{[22]}$ Becke's 1988 gradient correction, ${ }^{[23]}$ and HartreeFock exchange. Its correlation part, LYP, is the gradient-corrected functional of Lee, Yang, and Parr. ${ }^{[24]}$ The basis set used was Pople's double zeta split basis set with polarization functions $(6-31 \mathrm{~g} *) .{ }^{[25]}$ Ground state geometries were optimized using analytic gradients, conjugate gradient methods and the standard convergence criteria from Gaussian 09. ${ }^{[26]}$ From a single point calculation on the ground state geometry, the frontier orbital energies were deduced. To obtain values typical of the polymer, the calculations were carried out on oligomers of increasing length until the values of the frontier orbitals change less than 0.05 $\mathrm{eV}$. It was found that, because of the length of the monomers studied, two repeats unit were sufficient to achieve this. In order to obtain the optical band gaps for the polymers, nonadiabatic linear response calculations ${ }^{[27]}$ were carried out using the time dependent DFT formalism, as implemented in Gaussian 09, using the same density functional as basis set used in the geometry optimizations. These computations were carried out on the ground state geometries and are therefore an approximation to the absorption of the materials. The band gaps were computed using the time dependent formalism and the same functional and basis 


\section{Submitted to}

set. In order to obtain a theoretical value for the LUMO of fullerene, the same methods and basis sets were used; a theoretical value for the $\mathrm{PC}_{61} \mathrm{BM}$ LUMO of $-3.1 \mathrm{eV}$ was found. All computations were carried out using Gaussian 09.

\section{Results}

Figure 2 shows a contour plot of the maximum efficiencies possible as a function of the HOMO of the donor and its band gap. The main message from this model is clear: high efficiencies (in excess of 10\%) are simply not accessible unless polymers with a band gap of less than $2.1 \mathrm{eV}$ (more than $600 \mathrm{~nm}$ ) are utilized. Furthermore, even if small band gaps are obtained, the range of acceptable HOMO values becomes narrower and narrower. The key synthetic effort in this study has been concentrated on reducing the band gap of IF-TAA $(\mathrm{HOMO}=-5.5 \mathrm{eV}, \mathrm{Eg}=2.8 \mathrm{eV})$ whilst keeping its HOMO level approximately the same or slightly raised (-5.2 to $-5.3 \mathrm{eV})$ and maintaining its backbone architecture to ensure materials with a high charge carrier mobility.

In order to narrow the band gap of IF-TAA, the triarylamine unit is initially replaced with an acceptor unit (benzothiadiazole, BT) to afford a fully conjugated, more planar, copolymer IFBT $^{[28]}$ (Figure 1) which also exhibits a modest donor-acceptor (push-pull) type hybridization. As shown in Table 1 and Figure 3, this reduces the optical band gap as expected, due to a combination of molecular orbital hybridization and enhanced conjugation, but only to $2.3 \mathrm{eV}$ $(530 \mathrm{~nm})$, which is still too wide for highly efficient power conversion. A further improvement in band gap can be obtained by replacing the BT unit with the dithienobenzothiadiazole (TBTT) unit. ${ }^{[28]}$ This polymer (IF-TBTT, Figure 1) has indeed a narrower band gap of approximately $1.9 \mathrm{eV}(650 \mathrm{~nm}$, Figure 3). Effectively, the introduction of the two electron-rich thiophene units has increased the molecular orbital hybridization and raised the HOMO level. 


\section{Submitted to MATERIALS}

What are the structural features determining the band gap in these indenofluorene copolymers? A key feature of both the structures discussed so far is that they have a twisted backbone, due to unfavorable steric interactions between the $\alpha$-hydrogens (relative to the linking carbon atoms)of the indenofluorene unit and the neighboring co-monomer units. Eliminating any phenyl-phenyl links is therefore advantageous to ensuring aromatic coplanarity and extended conjugation, leading to reduced band gaps. The calculated torsion angles caused by these steric interactions are listed in Table 1 for all systems. Going from IFTAA to IF-BT preserves the unfavorable phenyl-phenyl links and hence a rather large torsional twist; the enhanced conjugation is caused by the removal of the $\mathrm{sp}^{3}$-hybridised nitrogen link. Next, comparing IF-TBTT to IF-BT, the phenyl-phenyl links are removed by the insertion of thiophene units and the backbone twist is reduced from $35^{\circ}$ to $23^{\circ}$, which explains the enhanced conjugation (along with the enhanced hybridization mentioned above). The next step to further reduce the band gap is to design structures that afford greater hybridization between the donor and acceptor moieties. Introducing thiophene rings next to the BT unit, as in the case of IF-TBTT, created a strong hybridization between the electron rich thiophene and the electron poor BT. However, in this structure, the weakly electrondonating IF unit is not really participating in the HOMO-LUMO molecular orbitals, and so to further improve the efficiency of the design, the IF unit was replaced with indacenodithiophene (IDT) to form IDT-BT (Figure 1). ${ }^{[29,30]}$ Similarly to IF, this repeat unit consists of three aromatic rings bridged into a rigid backbone by two sp3-hybridised carbon atoms. In the case of IF, the three aromatic rings are all benzenes, whereas the central benzene ring of IDT is flanked by two thiophene units. Our DFT calculations show that the thiophene functionalities have two geometric effects: i) they decrease steric hindrance due to reduced hydrogen-hydrogen interactions (calculated torsion angle is reduced to $7.4^{\circ}$ ), ii) they increase the electron-donating character of the monomer unit and hence ensure that the polymer has a more quinoidal form (stronger donor-acceptor hybridization), resulting in shorter bond 
distances between the IDT and the BT units (the calculated bond length is $1.45 \AA$ for IDT-BT and $1.48 \AA$ for IF-BT). The expected red-shifted absorption when compared to IF-TBTT is confirmed by UV-vis spectroscopy (Table 1, Figure 3) and we find an almost ideal band gap of $1.7 \mathrm{eV}(720 \mathrm{~nm})$ for IDT-BT. This step-wise lowering of the experimentally determined band gap from $2.8 \mathrm{eV}$ for IF-TAA to $1.7 \mathrm{eV}$ for IDT-BT with interim values of $2.3 \mathrm{eV}$ for IF-BT and $1.9 \mathrm{eV}$ for IF-TBTT is very nicely matched with the band gap values derived from the time-dependent DFT calculations as illustrated in Table 1; especially the narrow band gap materials show a very strong correlation between modeled and experimental data with discrepancies less than $0.1 \mathrm{eV}$.

The HOMO value of IF-TAA, as measured by photoelectron spectroscopy in air (PESA), is $5.5 \mathrm{eV}$. Introduction of the electron-poor BT unit lowers the HOMO significantly to $-5.9 \mathrm{eV}$ as expected. When going from IF-BT to IF-TBTT, the electron-rich thiophene units are anticipated to raise the HOMO, which is again verified experimentally with a value of $-5.6 \mathrm{eV}$. The last step of structural optimization, introduction of the IDT unit, is expected to raise the HOMO further due to the more electron-rich character of the monomer unit and the enhanced planarity along the backbone; an experimental HOMO value of $-5.3 \mathrm{eV}$ for IDT-BT confirms this assumption. Once again, we find the trend for the computed HOMO energy levels (Table 1) within this series of polymers to match very closely with the experimental data, although the actual values differ to a greater extent $(0.65-0.75 \mathrm{eV})$ than what was found for the band gaps. This large systematic shift in orbital energies is to be expected, as the DFT calculations are carried out in the vacuum and completely ignore dielectric solvation in the solid state. The shift also explains why, when we use HOMOs from DFT, it is important to match with LUMOs computed using similar methods.

Figure 4 depicts the calculated HOMO and LUMO distributions for a repeat unit of each polymer. IF-TAA, due to the electron-rich TAA unit, has its HOMO quite localized on the TAA unit (extending slightly onto the IF unit), whereas the LUMO correspondingly is located 
Submitted to MATERIALS

mainly on the less electron-rich IF unit. For the other three polymers, the introduction of the electron-poor BT unit and the resulting formation of strong donor-acceptor type structures change the HOMO/LUMO distributions significantly. The HOMOs are now completely delocalized along the entire conjugated backbone for IF-BT, IF-TBTT and IDT-BT, while the LUMOs are strongly localized on the BT acceptor unit. We also note a slight participation of the IDT unit in the LUMO of IDT-BT, which supports our hypothesis of a stronger donoracceptor hybridization and hence a more quinoidal form for this polymer than for the IF-based polymers.

From a purely theoretical approach, applying the maximum efficiency model to the HOMO levels and band gaps derived from DFT calculations, we find that IF-TAA holds very poor potential for OPV applications mainly due to its very wide band gap. IF-BT should perform moderately well with a predicted efficiency of $6 \%$, while both IF-TBTT and IDT-BT are predicted to be superior at $11 \%$ and $13 \%$ maximum efficiency (Table 2) predominantly due to their narrower band gaps. As discussed above, while the DFT calculations quite precisely have predicted the band gaps of these polymers, a significant discrepancy is observed between theoretical and experimental HOMO values for the series. Therefore, we have also applied the maximum efficiency model to the HOMO levels and band gaps extracted from our experimental characterization of these polymers. As depicted in Figure 2, IF-TAA is still predicted to perform poorly in a solar cell (1.8\% PCE), while IF-BT now has a calculated maximum efficiency of 7.5\%. Quite significant increases in OPV performance are again anticipated when going to IF-TBTT (12\%) and subsequently to IDT-BT reaching a predicted maximum efficiency of $13.5 \%$.

It is obvious that the output from the two models is different in terms of absolute values, but at the same time, it is clear that IF-TBTT and IDT-BT in both instances are predicted to perform better than IF-BT in an OPV device; the main reason being the gradual narrowing of the band gap. This predicted trend in OPV performance is verified experimentally as 
highlighted in Table 3. Whereas IF-TAA and IF-BT both have negligible power conversion efficiencies due to their wide band gaps, IF-TBTT shows a PCE of $2.0 \%$ in a solar cell and IDT-BT reaches a maximum PCE of $5.5 \% . .^{[28,30]}$ On comparison of IF-TBTT and IDT-BT, we furthermore notice a good agreement between the measured OPV parameters $\left(\mathrm{V}_{\mathrm{oc}}\right.$ and $\left.\mathrm{J}_{\mathrm{sc}}\right)$ and the energy levels. The lower-lying HOMO of IF-TBTT results, as expected, in a higher Voc as compared to IDT-BT. At the same time, and more significantly, the narrower band gap of IDT-BT is matched with a higher $\mathbf{J}_{\mathrm{sc}}$, which ultimately results in a better device performance as compared to IF-TBTT. As also mentioned in the introduction, it is worth highlighting again that the realization of optimum HOMO and LUMO energy levels is far from the only obstacle towards efficient BHJ polymer solar cells. As is evident from the three entries for IDT-BT in Table 3, device performance depends strongly on the morphology of the blend, which can be tuned not only by side-chain modifications as seen here, but also by solvent effects, additives, and processing and annealing conditions. Recent literature has furthermore indicated that device performance can be further optimized through electrode/interlayer modifications ${ }^{[31,32]}$ results which can obviously contribute to narrowing the gap between theoretical and experimental OPV device performances.

As mentioned in the introduction, the rigid indenofluorene unit tends to promote good charge carrier mobility, which is a prerequisite for good performance in a photovoltaic device. IFTAA displayed a hole mobility of $0.04 \mathrm{~cm}^{2} / \mathrm{Vs}$ despite its rather large torsional twist and amorphous microstructure. ${ }^{[19]}$ Zheng and co-workers found IF-TBTT to have a similar hole mobility of $0.01 \mathrm{~cm}^{2} / \mathrm{Vs}{ }^{[33]}$ Two opposing effects seem to play a role when comparing these two polymers: one the one hand, the TAA unit is well-known as a good hole transporter, and on the other hand, the TBTT unit promotes backbone coplanarity and extended conjugation crucial for good charge transport. Further planarization of the backbone and increased orbital hybridization turns out to be hugely favorable for the charge transport. In an OFET device, IDT-BT reaches a maximum hole mobility of $1.2 \mathrm{~cm}^{2} / \mathrm{Vs} .{ }^{[29]}$ If considered as a screening tool 
during the design and synthesis of novel polymeric materials for OPV applications, it is obvious that the polymers presented in this study all possess adequate charge transport properties.

\section{Conclusion}

In conclusion, we have applied a systematic approach to the structural optimization of IFbased polymers for organic photovoltaics. A successful outcome of this process is evident as the frontier energy levels has been gradually adjusted and optimized for efficient BHJ solar cells with PCBM as the electron acceptor. Starting from IF-TAA, the first step in the design optimization was to introduce the electron-poor BT unit to significantly narrow the band gap as a consequence of donor-acceptor mediated hybridization. Secondly, a further improvement in terms of a smaller band gap (the result of a raised HOMO level) was realized when going from IF-BT to IF-TBTT. Finally, introduction of the more electron-rich IDT unit was found to further raise the HOMO level and hence reduce the band gap favorably. Arriving at IDTBT as our optimized structure with ideal frontier energy levels has allowed us to fabricate highly efficient OPV devices as well as excellent OFET devices. Other IF- and IDT-based donor-acceptor type copolymers reported in the literature appear to have frontier energy levels very similar to IDT-BT and it seems like the rational design strategy aimed at the optimization of indenofluorene polymers for organic photovoltaics has been pursued very far towards the limit of what can be achieved with this class of materials. ${ }^{[33-36,37]}$

To further prove the strength of a rational design optimization approach, we have conducted a thorough computational study of this series of polymers, which clearly support the experimental findings. The maximum efficiency model presented herein accurately predicts the potential of IF-TBTT and IDT-BT for use in organic photovoltaics, while IF-TAA and IF-BT similarly are predicted to have poorly located frontier energy levels that make these materials unsuitable for highly efficient OPV devices. The potential aid in structural 
Submitted to MATERIALS

optimization from quantum-chemical models like this should not be underestimated; it is clear that the theoretically obtained efficiencies, especially when used in conjunction with initial experimental characteristics, are good indicators that can be used in the initial screening process when developing new materials for the continued development of organic photovoltaics.

\section{Acknowledgements}

This work was in part carried out with financial support from SUPERGEN, EC FP7 Project X10D and EC FP7 Project ONE-P, with support from the Centre for Plastic Electronics at Imperial College and the International Collaborative Research Program of Gyeonggi-do, Korea. JK is a member of the Oxford Centre for Collaborative Applied Mathematics (OCCAM) where his work is supported by Award No. KUK-C1-013-04, made by King Abdullah University of Science and Technology.

Received: ((will be filled in by the editorial staff))

Revised: ((will be filled in by the editorial staff)) Published online: ((will be filled in by the editorial staff))

[1] Hains, A. W.; Liang, Z.; Woodhouse, M. A.; Gregg, B. A. Chem. Rev. 2010, 110, 6689.

[2] Facchetti, A. Chem. Mater. 2011, 23, 733.

[3] Chochos, C. L.; Choulis, S. A. Prog. Polym. Sci. 2011, 36, 1326.

[4] Brabec, C. J.; Gowrisanker, S.; Halls, J. J. M.; Laird, D.; Jia, S.; Williams, S. P. Adv. Mater. 2010, 22, 3839.

[5] Thompson, B. C.; Fréchet, J. M. J. Angew. Chem. Int. Ed. 2008, 47, 58.

[6] Nelson, J.; Kirkpatrick, J.; Ravirajan, P. Phys. Rev. B 2004, 69, 035337.

[7] Ma, W.; Yang, C.; Gong, X.; Lee, K.; Heeger, A. J. Adv. Funct. Mater. 2005, 15, 1617.

[8] Sivula, K.; Luscombe, C. K.; Thompson, B. C.; Fréchet, J. M. J. J. Am. Chem. Soc. 2006, 128, 13988. 
Submitted to MATERIALS

[9] Woo, C. H.; Thompson, B. C.; Kim, B. J.; Toney, M. F.; Fréchet, J. M. J. J. Am. Chem. Soc. 2008, 130, 16324.

[10] Dang, M. T.; Hirsch, L.; Wantz, G. Adv. Mater. 2011, 23, 3597.

[11] Liang, Y.; Feng, D.; Wu, Y.; Tsai, S.-T.; Li, G.; Ray, C.; Yu, L. J. Am. Chem. Soc. 2009, 131, 7792 .

[12] Piliego, C.; Holcombe, T. W.; Douglas, J. D.; Woo, C. H.; Beaujuge, P. M.; Fréchet, J. M. J. J. Am. Chem. Soc. 2010, 132, 7595.

[13] Ballantyne, A. M.; Chen, L.; Dane, J.; Hammant, T.; Braun, F. M.; Heeney, M.; Duffy, W.; McCulloch, I.; Bradley, D. D. C.; Nelson, J. Adv. Funct. Mater. 2008, 18, 2373.

[14] Müller, C.; Wang, E.; Andersson, L. M.; Tvingstedt, K.; Zhou, Y.; Andersson, M. R.; Inganäs, O. Adv. Funct. Mater. 2010, 20, 2124.

[15] Hoppe, H.; Niggemann, M.; Winder, C.; Kraut, J.; Hiesgen, R.; Hinsch, A.; Meissner, D.; Sariciftci, N. S. Adv. Funct. Mater. 2004, 14, 1005.

[16] Lee, J. K.; Ma, W. L.; Brabec, C. J.; Yuen, J.; Moon, J. S.; Kim, J. Y.; Lee, K.; Bazan, G. C.; Heeger, A. J. J. Am. Chem. Soc. 2008, 130, 3619.

[17] Liang, Y.; Xu, Z.; Xia, J.; Tsai, S.-T.; Wu, Y.; Li, G.; Ray, C.; Yu, L. Adv. Mater. 2010, 22, E135.

[18] Scharber, M. C.; Mühlbacher, D.; Koppe, M.; Denk, P.; Waldauf, C.; Heeger, A. J.; Brabec, C. J. Adv. Mater. 2006, 18, 789.

[19] Zhang, W.; Smith, J.; Hamilton, R.; Heeney, M.; Kirkpatrick, J.; Song, K.; Watkins, S. E.; Anthopoulos, T.; McCulloch, I. J. Am. Chem. Soc. 2009, 131, 10814.

[20] Frost, J. M.; Faist, M. A.; Nelson, J. Adv. Mater. 2010, 22, 4881.

[21] Becke, A. D. J. Chem. Phys. 1993, 98, 5648.

[22] Slater, J. C. Quantum Theory of Molecules and Solids; McGraw- Hill: New York, 1974.; Vol. 4.

[23] Becke, A. D. Phys. Rev. A 1988, 38, 3098. 
Submitted to

[24] Lee, C.; Yang, W.; Parr, R. G. Phys. Rev. B 1988, 37, 785.

[25] Ditchfield, R.; Hehre, W. J.; Pople, J. A. J. Chem. Phys. 1971, 54, 724.

[26] M. J. Frisch, G. W. Trucks, H. B. Schlegel, G. E. Scuseria, M. A. Robb, J. R.

Cheeseman, G. Scalmani, V. Barone, B. Mennucci, G. A. Petersson, H. Nakatsuji, M.

Caricato, X. Li, H. P. Hratchian, A. F. Izmaylov, J. Bloino, G. Zheng, J. L. Sonnenberg, M.

Hada, M. Ehara, K. Toyota, R. Fukuda, J. Hasegawa, M. Ishida, T. Nakajima, Y. Honda, O.

Kitao, H. Nakai, T. Vreven, J. A. Montgomery, Jr., J. E. Peralta, F. Ogliaro, M. Bearpark, J. J.

Heyd, E. Brothers, K. N. Kudin, V. N. Staroverov, R. Kobayashi, J. Normand, K.

Raghavachari, A. Rendell, J. C. Burant, S. S. Iyengar, J. Tomasi, M. Cossi, N. Rega, J. M.

Millam, M. Klene, J. E. Knox, J. B. Cross, V. Bakken, C. Adamo, J. Jaramillo, R. Gomperts,

R. E. Stratmann, O. Yazyev, A. J. Austin, R. Cammi, C. Pomelli, J. W. Ochterski, R. L.

Martin, K. Morokuma, V. G. Zakrzewski, G. A. Voth, P. Salvador, J. J. Dannenberg, S.

Dapprich, A. D. Daniels, O. Farkas, J. B. Foresman, J. V. Ortiz, J. Cioslowski, and D. J. Fox, Gaussian 09, Revision A.02, Gaussian, Inc., Wallingford CT, 2009.

[27] Bauernschmitt, R.; Ahlrichs, R. Chem. Phys. Lett. 1996, 256, 454.

[28] Soon, Y. W.; Clarke, T. M.; Zhang, W.; Agostinelli, T.; Kirkpatrick, J.; Dyer-Smith,

C.; McCulloch, I.; Nelson, J.; Durrant, J. R. Chem. Sci. 2011, 2, 1111.

[29] Zhang, W.; Smith, J.; Watkins, S. E.; Gysel, R.; McGehee, M.; Salleo, A.; Kirkpatrick, J.; Ashraf, S.; Anthopoulos, T.; Heeney, M.; McCulloch, I. J. Am. Chem. Soc. 2010, 132, 11437.

[30] Bronstein, H.; Leem, D. S.; Hamilton, R.; Woebkenberg, P.; King, S.; Zhang, W.; Ashraf, R. S.; Heeney, M.; Anthopoulos, T. D.; Mello, J. D.; Mcculloch, I. Macromolecules 2011, 6649.

[31] He, Z.; Zhang, C.; Xu, X.; Zhang, L.; Huang, L.; Chen, J.; Wu, H.; Cao, Y. Adv. Mater. 2011, 23, 3086. 
[32] He, Z.; Zhong, C.; Huang, X.; Wong, W.-Y.; Wu, H.; Chen, L.; Su, S.; Cao, Y. Adv.

Mater. 2011.

[33] Zheng, Q.; Jung, B. J.; Sun, J.; Katz, H. E. J. Am. Chem. Soc. 2010, 132, 5394.

[34] Zhang, M.; Guo, X.; Wang, X.; Wang, H. Chem. Mater. 2011, 23, 4264.

[35] Zhang, Y.; Zou, J.; Yip, H.-L.; Chen, K.-S.; Zeigler, D. F.; Sun, Y.; Jen, A. K. Y.

Chem. Mater. 2011, 23, 2289.

[36] Sun, Y.; Chien, S.-C.; Yip, H.-L.; Zhang, Y.; Chen, K.-S.; Zeigler, D. F.; Chen, F.-C.;

Lin, B.; Jen, A. K.-Y. J. Mater. Chem. 2011, 21, 13247.

[37] Ashraf, R. S.; Chen, Z.; Leem, D. S.; Bronstein, H.; Zhang, W.; Schroeder, B.; Geerts, Y.; Smith, J.; Watkins, S.; Anthopoulos, T. D.; Sirringhaus, H.; de Mello, J. C.; Heeney, M.;

McCulloch, I. Chem. Mater. 2011, 23, 768. 
<smiles>[R]C1([R])c2cc(C)ccc2-c2cc3c(cc21)-c1ccc(-c2ccc(N(c4ccc(C)cc4)c4ccc(C)cc4C)cc2)cc1C3([R])[R]</smiles><smiles>[R]C1([R])c2cc(C)ccc2-c2cc3c(cc21)-c1ccc(-c2ccc(-c4ccc(-c5ccc(C(C)(C)C)s5)c5nsnc45)s2)cc1C3([R])[R]</smiles>

IF-TBTT<smiles>[R]C1([R])c2cc(C)ccc2-c2cc3c(cc21)-c1ccc(-c2ccc(C)c4nsnc24)cc1C3([R])[R]</smiles>

IF-BT

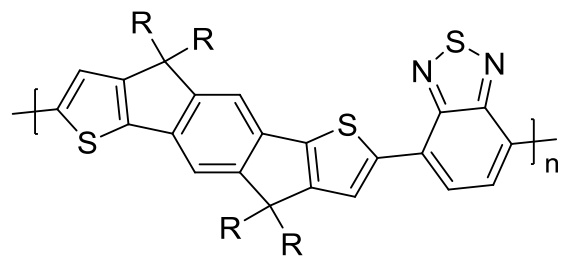

IDT-BT

Figure 1. The chemical structures of the polymers discussed in this study.

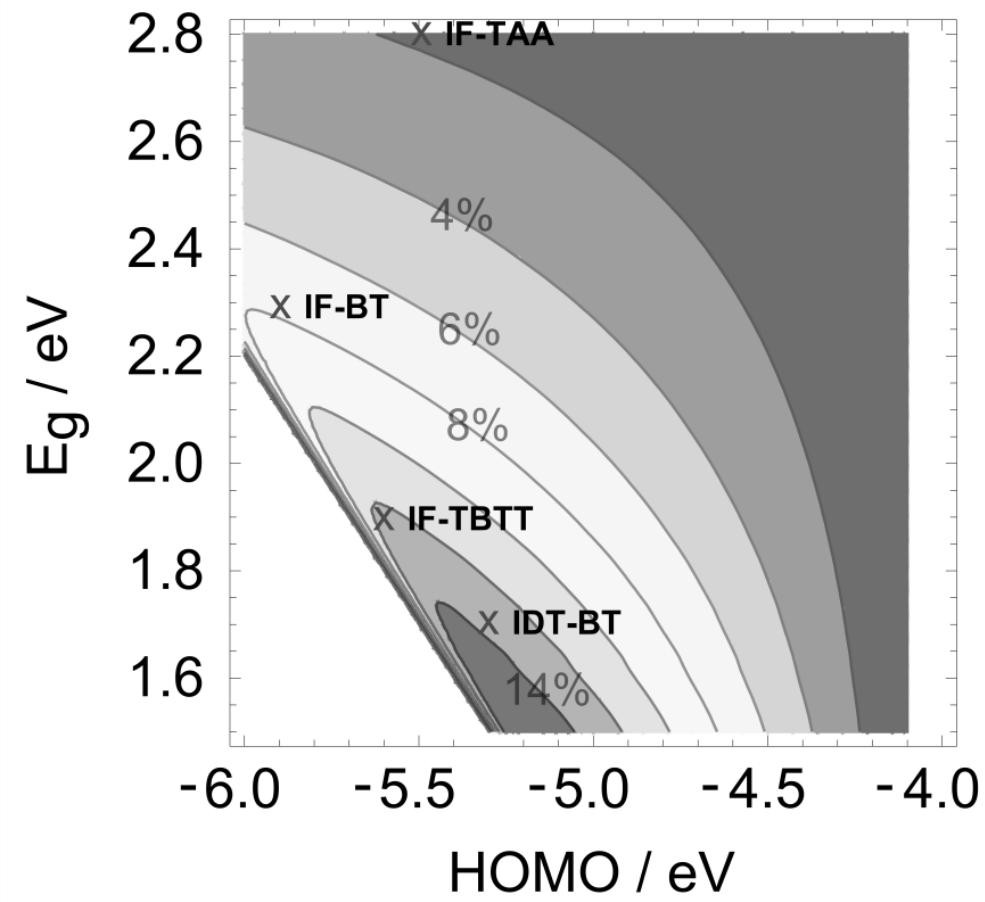

Figure 2. Contour plot depicting the maximum OPV power conversion efficiencies possible as a function of the donor material's HOMO level and band gap (a constant fill factor of 0.65 has been assumed). Predicted efficiencies based on experimental energy levels for the four polymers are marked with crosses. 
Submitted to MATERIALS

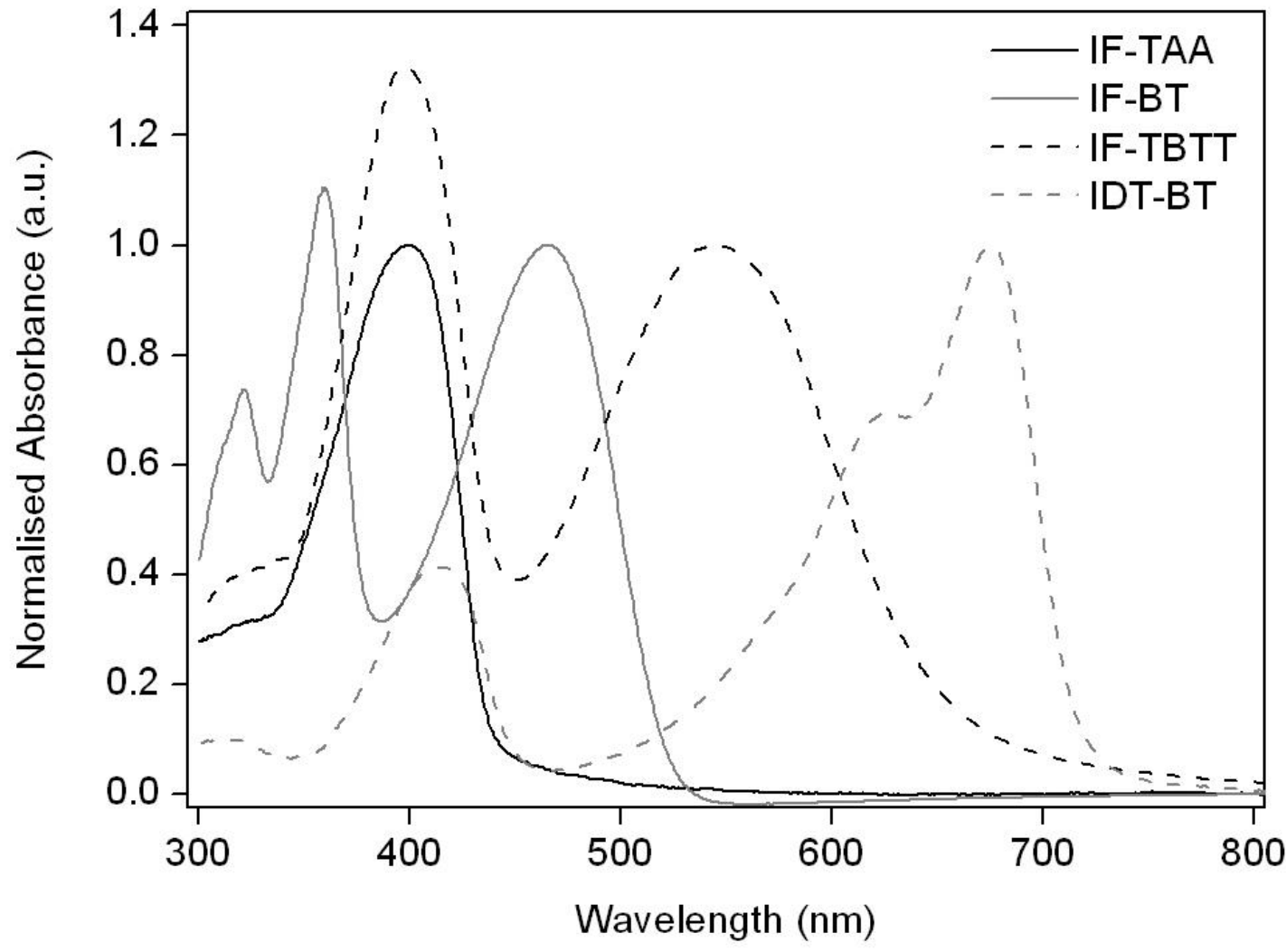

Figure 3. UV-vis spectra in the solid state of the four polymers discussed in this study. 


\section{ADVANCED \\ ENERGY \\ Submitted to MATERIALS}
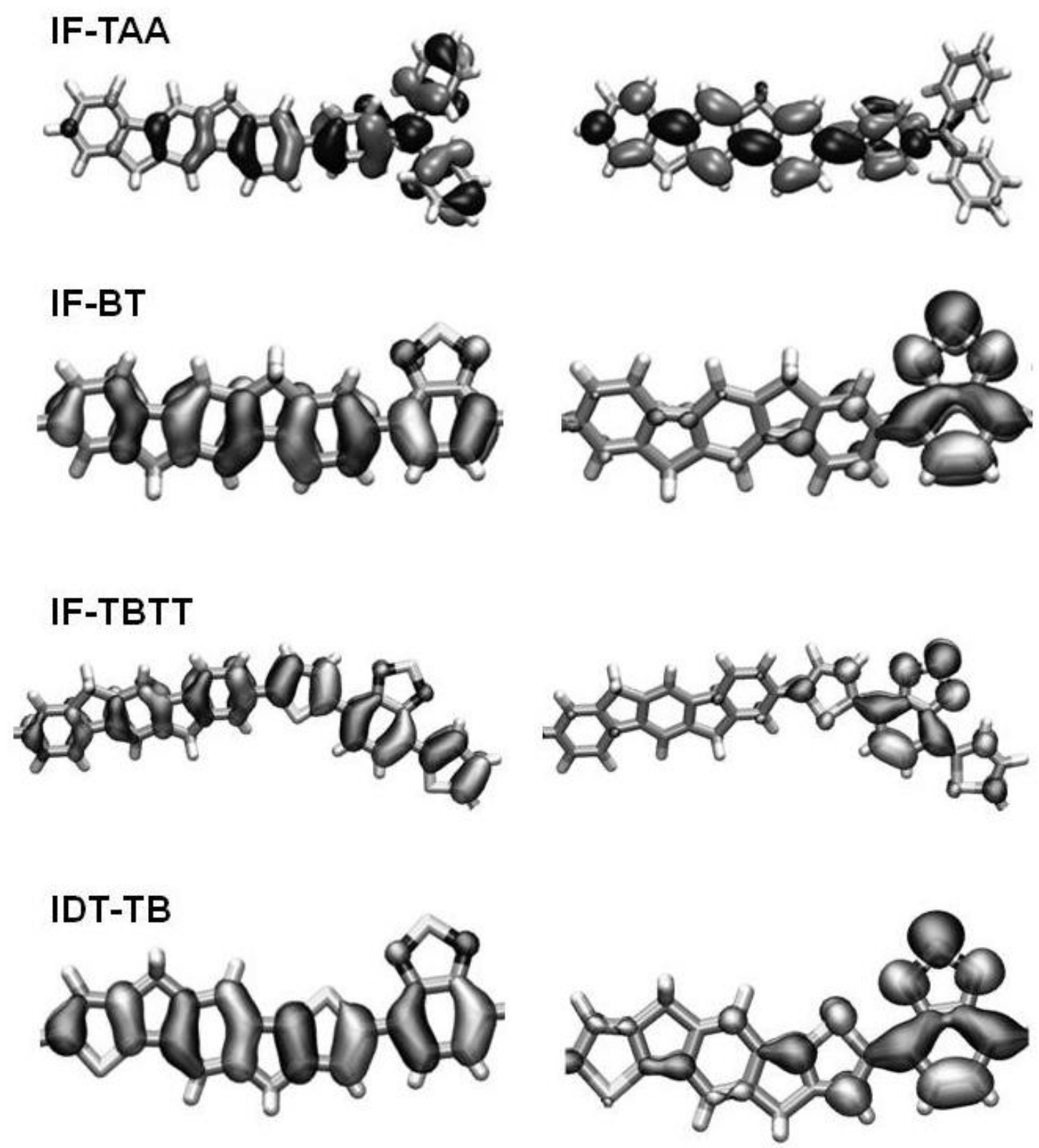

Figure 4. HOMO (left) and LUMO (right) distributions for the minimum-energy conformations of the investigated polymers optimized with Gaussian at the B3LYP/6-31G* level. 
Table 1. Experimental and computational energy levels of the studied polymers.

\begin{tabular}{lccccccc}
\hline Material & $\mathrm{R}$ & $\mathrm{M}_{\mathrm{n}} / \mathrm{M}_{\mathrm{w}}$ & $\begin{array}{c}\lambda_{\max } \\
(\mathrm{UV}-\mathrm{vis}) \\
{[\mathrm{nm}]}\end{array}$ & $\begin{array}{c}\mathrm{HOMO} \\
(\mathrm{DFT}) \\
{[\mathrm{kg} / \mathrm{mol}]}\end{array}$ & $\begin{array}{c}\text { HOMO } \\
(\mathrm{PESA}) \\
{[\mathrm{eV}]}\end{array}$ & $\begin{array}{c}\mathrm{S}_{1} \\
(\mathrm{TDDFT}) \\
{[\mathrm{eV}]}\end{array}$ & $\begin{array}{c}\mathrm{S}_{1} \\
(\mathrm{UV}-\mathrm{vis}) \\
{[\mathrm{eV}]}\end{array}$ \\
\hline IF-TAA & 1 -octyl & $15 / 25$ & 399 & -4.75 & -5.5 & 3.00 & 2.8 \\
IF-BT & 1 -octyl & $15 / 38$ & 465 & -5.15 & -5.9 & 2.37 & 2.3 \\
IF-TBTT & 1 1-octyl & $13 / 27$ & 545 & -4.88 & -5.6 & 1.92 & 1.9 \\
IDT-BT & 1 1-octyl & $33 / 243$ & 674 & -4.65 & -5.3 & 1.74 & 1.7 \\
\hline
\end{tabular}

Table 2. Computational data for the studied polymers.

\begin{tabular}{lccc}
\hline Material & $\begin{array}{c}\text { Torsion angle } \\
{\left[{ }^{\circ}\right]}\end{array}$ & $\begin{array}{c}\operatorname{Max} \eta^{\text {a) }} \\
{[\%]}\end{array}$ & $\begin{array}{c}\operatorname{Max} \eta^{\text {b) }} \\
{[\%]}\end{array}$ \\
\hline IF-TAA & 35 & 0.9 & 1.8 \\
IF-BT & 35 & 6.1 & 7.5 \\
IF-TBTT & 23 & 11.0 & 12.0 \\
IDT-BT & 7.4 & 13.0 & 13.5
\end{tabular}

${ }^{\text {a) }}$ Maximum efficiencies calculated from DFT energy levels; ${ }^{\text {b) }}$ maximum efficiencies calculated from experimental energy levels.

Table 3. Experimental OPV parameters for the studied polymers.

\begin{tabular}{lcccccc}
\hline Material & $\mathrm{R}$ & $\begin{array}{c}\mathrm{M}_{\mathrm{n}} / \mathrm{M}_{\mathrm{w}} \\
{[\mathrm{kg} / \mathrm{mol}]}\end{array}$ & $\begin{array}{c}\mathrm{V}_{\mathrm{oc}} \\
{[\mathrm{V}]}\end{array}$ & $\begin{array}{c}\mathrm{J}_{\mathrm{sc}} \\
{\left[\mathrm{mA} / \mathrm{cm}^{2}\right]}\end{array}$ & $\mathrm{FF}$ & $\begin{array}{c}\text { PCE } \\
{[\%]}\end{array}$ \\
\hline IF-TAA $^{\text {a) }}$ & 1 -octyl & $15 / 25$ & - & - & - & - \\
IF-BT $^{\text {a) }}$ & 1 -octyl & $15 / 38$ & - & - & - & - \\
IF-TBTT $^{\text {b) }}$ & 1 -octyl & $13 / 27$ & 0.97 & 4.86 & 0.43 & 2.05 \\
IDT-BT $^{\text {c) }}$ & $\begin{array}{c}1 \text {-octyl } \\
1-(2-\end{array}$ & $33 / 243$ & 0.74 & 10.05 & 0.46 & 3.39 \\
IDT-BT $^{\text {c) }}$ & $\begin{array}{c}\text { ethylhexyl) } \\
\text { (2) }\end{array}$ & $40 / 87$ & 0.79 & 13.0 & 0.54 & 5.50 \\
\hline
\end{tabular}

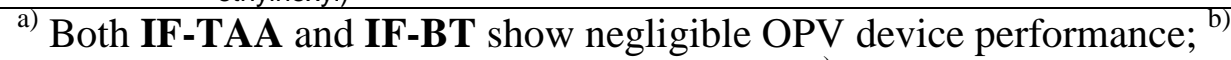
ITO/PEDOT:PSS/IF-TBTT:PC ${ }_{61} \mathrm{BM}(1: 4) / \mathrm{Al} ;{ }^{\mathrm{c})}$ ITO/PEDOT:PSS/IDT-BT:PC ${ }_{71} \mathrm{BM}$ $(1: 3.5) / \mathrm{Ca} / \mathrm{Al}$. 
A series of fused aromatic push-pull copolymers for organic solar cells is presented. A retrospective analysis based on molecular calculations affords good estimates of the experimental absorption energies and ionization potentials. Comparing photovoltaic device performances of the polymer series to the trend in efficiencies predicted by computation confirms the validity of this approach.

Keywords conjugated polymers; DFT calculations; molecular simulations; organic photovoltaics; solar cells

James Kirkpatrick, * Christian B. Nielsen,* Weimin Zhang, Hugo Bronstein, R. Shahid Ashraf, Martin Heeney, and Iain McCulloch

A Systematic Approach to the Design Optimization of Light Absorbing Indenofluorene Polymers for Organic Photovoltaics

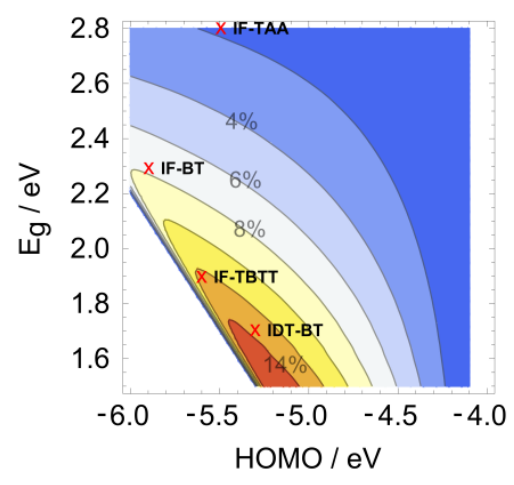

